Article

\title{
Application of Bacteriophages to Control Vibrio alginolyticus Contamination in Oyster (Saccostrea glomerata) Larvae
}

\author{
Tuan Son Le ${ }^{1,2}$, Paul C. Southgate ${ }^{3}$, Wayne $\mathrm{O}^{\prime}$ Connor $^{4}$, Sang V. Vu ${ }^{2}$ and D. İpek Kurtböke ${ }^{2, *(D)}$ \\ 1 Research Institute for Marine Fisheries, 224 Le Lai, Ngo Quyen, Hai Phong 180000, Vietnam; \\ letuanson1987@gmail.com or Tuan.Son.Le@research.usc.edu.au \\ 2 GeneCology Research Centre and School of Science and Engineering, University of the Sunshine Coast, \\ 90 Sippy Downs Drive, Sippy Downs, Queensland 4556, Australia; vuvansangts50@gmail.com or \\ v_v013@student.usc.edu.au \\ 3 Australian Centre for Pacific Islands Research and School of Science and Engineering, University of the \\ Sunshine Coast, Maroochydore, Queensland 4556, Australia; psouthgate@usc.edu.au \\ 4 NSW Fisheries, Port Stephens Fisheries Institute, Taylors Beach 2316, Australia; \\ wayne.oconnor@dpi.nsw.gov.au \\ * Correspondence: ikurtbok@usc.edu.au; Tel.: +61-7-5430-2918
}

Received: 5 June 2020; Accepted: 9 July 2020; Published: 16 July 2020

check for updates

\begin{abstract}
Mortalities of bivalve larvae and spat linked with Vibrio spp. infection have been described in hatcheries since 1959, causing potential development of resistant bacteria. A reliable and sustainable solution to this problem is yet to be developed. Potential treatment of bacterial infection with bacteriophages is gaining interest in aquaculture as a more sustainable option for managing Vibrio spp. infection. This study assessed the effectiveness of bacteriophages $(\Phi-5, \Phi-6$, and $\Phi-7)$ against pathogenic Vibrio isolates (USC-26004 and USC-26005). These phage isolates were found to belong to the Myoviridae viral family. A total of 212 ORFs of $\Phi-5$ were identified and annotated. The genome of this phage contained putative thymidine kinase and lysin enzyme. During infections with phages, the OD values of the isolates USC-26005 and USC-26004 remained stable at a much lower reading compared to the control after $9 \mathrm{~h}$ of incubation. Mortality rate of oyster (Saccostrea glomerata) larvae was $28.2 \pm 3.5 \%$ in the bacteriophage treatment group, compared to $77.9 \pm 9.1 \%$ in the bacterial treatment group after $24 \mathrm{~h}$ incubation. Findings of this study indicate that lytic phages might be utilized as potential bio-control agents of luminescent bacterial disease in oyster hatcheries.
\end{abstract}

Keywords: Vibrio alginolyticus; vibriosis; bacteriophage; biocontrol; oyster larvae

\section{Introduction}

Global oyster production accounted for 31\% of total world mollusk production in 2016 [1] and a significant proportion of this output relied on hatchery production [2]. However, bivalve hatcheries are constantly threatened by bacterial diseases [3] and in most cases, members of the genus Vibrio have been identified as significant pathogens in production environments [4]. Since the first description of Vibrio species as the causative agents of disease in bivalve larvae [5], numerous studies have identified pathogenic Vibrio spp., including V. tubiashii [3], V. splendidus biovar II [6], and V. ostreicida [7] associated with infections that have caused major losses for shellfish farmers [8].

Major activities within oyster hatcheries include broodstock holding, spawning induction, rearing and setting of larvae, and the culture and production of live microalgae as a larval food source [2]. Three key vectors for potential Vibrio spp. contamination in these hatcheries include broodstock, microalgae cultures, and seawater supply $[9,10]$. 
Antibiotics have been used to treat vibriosis in aquaculture systems [11]; however, there are considerable concerns regarding the long-term use of antibiotics because of the potential for development of antibiotic-resistant bacteria [11], which could be exacerbated by transmission of antibiotic resistance genes into different members of hatchery microbiota by horizontal transfer mechanisms [10]. Karunasagar et al. [12] indicated that mass mortality of black tiger shrimp (Penaeus monodon) larvae was caused by multi-drug resistant $V$. harveyi strains with reported resistance to cotrimoxazole, chloramphenicol, erythromycin, and streptomycin. Similarly, the Vibrio spp. isolated from fish-pond facilities in Nigeria were resistant to tetracyclines (100\%), oxytetracycline (99.4\%), and chloramphenicol (73.1\%) [13]. Resistance to antibiotics by Vibrio spp. has also been reported in infected bivalve mollusks; examples include resistance to ampicillin, vancomycin, and erythromycin by 15 strains of $V$. alginolyticus [14]. In some countries, use of antibiotics in aquaculture species is now prohibited because of potential side effects and development of harmful residues [15].

Although mortalities of bivalve larvae and spat linked with Vibrio spp. infections have been described in hatcheries since 1959 [5], a reliable and sustainable solution to disease control is yet to be developed. Treatment of bacterial infections with bacteriophages is gaining considerable interest as a safer alternative for its potential to reduce or remove bacterial contamination in aquaculture systems [11]. Examples include bacteriophage control of $V$. parahyemolyticus infection in adult oysters (Ostrea plicatula) [16] and in striped catfish (Pangasianodon hypophthalmus) [17], and bacteriophage treatment of $V$. coralliilyticus infection in Pacific oyster (Crassostrea gigas) [18].

In addition, phage enzymes can have antibacterial activities. At the end of the lytic cycle of double-stranded DNA bacteriophages, holin, and lysin enzymes are created in order for bacteriophages to exit the infected bacterial host cell. With the help of holins which form large and non-selective pores in the cytoplasmic membrane, lysins can interact with the peptidoglycan substrate and cause cell lysis [19]. The holin and lysin interaction is known as the lambda paradigm [20]. Lysin is considered to be one of the most effective anti-microbial agents because $\sim 50 \%$ of bacteria on the planet are killed by the bacteriophage lytic cycle every 2 days [21]. Lysins, which are greater than $25 \mathrm{kDa}$ in size, have modular organization defined by two distinct domains: N-terminal domain and C-terminal binding domain, corresponding to the two functions of enzymatic hydrolysis and substrate recognition. In general, bacteriophage lysins quickly break the cell wall of host bacteria, especially the Gram-positive bacteria. However, the access of lysins to the peptidoglycan in Gram-negative bacteria can be problematic because the outer membrane can block their entry and, as a result, their efficiency is limited [20]. Recently, more research has focused on lysin enzymes such as lysin Cpl-1 to treat pneumococcal infection [22,23], PlySs2 to treat Streptococcus pyogenes and Staphylococcus aureus [24]; PlyCD for Clostridium difficile [25]; PlyE146 for E. coli K12, Pseudomonas aeruginosa, and Acinetobacter baumannii [26]; PlyF307 for Acinetobacter baumannii [27]; and ABgp46 for Acinetobacter baumannii, Pseudomonas aeruginosa, and Salmonella typhimurium [28].

Development of bacteriophage biocontrol methods as a means of treating Vibrio spp. contamination in aquaculture systems is attractive for three main reasons: (1) Most bacteria are parasitized by at least one bacteriophage and the bacteriophage plays an important role in the evolution of the host genome; (2) land and water resources host large numbers of bacteriophages, estimated at 10-100 times greater than that of the host bacteria; and (3) the potential of bacteriophage therapy as an alternative treatment to antibiotics [29]. However, the effectiveness and potential application of bacteriophage therapy for reduction of Vibrio spp. in oyster hatcheries has not yet been extensively studied. The objectives of this study were, therefore, to isolate pathogenic Vibrio spp. and their specific bacteriophages, and to determine the effectiveness of a bacteriophage cocktail composed of these isolated bacteriophages against Vibrio spp. infesting cultures of oyster larvae. Moreover, characteristics of the isolated bacteriophages, including a full genome analysis, were also investigated. 


\section{Results}

\subsection{Vibrio spp. Isolation and Pathogenicity Test}

Conventional biochemical tests revealed that the isolates USC-26004 and USC-26005 were V. alginolyticus (98.2\%) and V. alginolyticus (98.6\%), respectively. The isolate USC-26004 (Figure 1) caused $100 \%$ mortality of oyster larvae within $36 \mathrm{~h}$ with a pathogenicity rate of $17.7 \%$. In contrast, the pathogenicity rate of USC-26005 was $2.5 \%$ (Table 1 ).
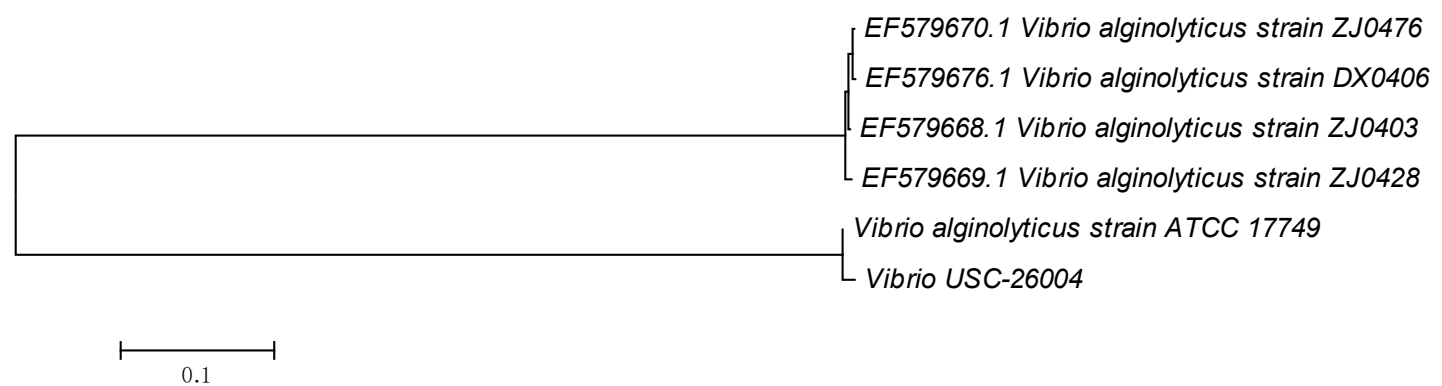

Figure 1. Phylogenetic tree of the pathogenic Vibrio sp. (USC-26004) in relation to its closest relatives.

Table 1. Recorded mortality (\%) of oyster (Saccostrea glomerata) larvae in challenge tests when exposed to different Vibrio isolates.

\begin{tabular}{ccc}
\hline Vibrio Species & BLAST (Identity \%) & Pathogenicity Rate (\%) \\
\hline USC-26004 & V. alginolyticus (99\%) & 17.7 \\
USC-26005 & V. alginolyticus (99\%) & 2.5 \\
ATCC 14126 & V. harveyi & 16.6 \\
ATCC 17749 & V. alginolyticus & 12.9 \\
\hline
\end{tabular}

Data presented as averages. All tested groups were performed in triplicate using the method of Prado et al. [30].

\subsection{Prophage Detection in Bacterial Isolates and Antibiotic Susceptibility}

The concentration of bacterial isolates increased over the incubation period. No plaque from the spot technique was seen (results not shown). Therefore, there was no prophage detected in the isolated Vibrio USC-26005 and USC-26004's chromosomes.

Vibrio isolate USC-26004 was found to be resistant to aztreonam, erythromycin, gentamicin, streptomycin, cefoxitin, and ampicillin and to have intermediate-resistance to imipenem.

\subsection{Isolation and Characterization of Bacteriophages}

Three different bacteriophages namely $\Phi-5, \Phi-6$, and $\Phi-7$ were isolated and examined using transmission electron microscope (TEM) which showed that all bacteriophages had long tails. Characteristics of these bacteriophages are shown in Figure 2 and Table 2.

Table 2. Characteristics of the Vibrio bacteriophages isolated in this study.

\begin{tabular}{|c|c|c|c|c|c|c|c|c|}
\hline \multirow{2}{*}{ Bacteriophage } & \multicolumn{2}{|c|}{ Head (nm) } & \multirow{2}{*}{ Nucleic Acid } & \multicolumn{2}{|c|}{ Neck (nm) } & \multicolumn{2}{|c|}{ Tail Sheath (nm) } & \multirow{2}{*}{ Family } \\
\hline & $\mathbf{L}$ & $\mathbf{W}$ & & $\mathbf{L}$ & $\mathbf{W}$ & $\mathbf{L}$ & $\mathbf{W}$ & \\
\hline$\Phi-5$ & 131.8 & 122.7 & DNA, ds & 11.4 & 6.8 & 129.5 & 18.2 & Myoviridae \\
\hline$\Phi-6$ & NM & 96.0 & DNA, ds & NM & NM & 162.2 & 16.1 & Myoviridae \\
\hline$\Phi-7$ & 68.6 & 59.1 & DNA, ds & 4.76 & 3.8 & 81.9 & 8.6 & Myoviridae \\
\hline
\end{tabular}

The concentration of bacteriophages was $\sim \times 10^{8}$ PFU/mL; W: wide; L: length, NM: not measured. Data presented as averages. Bacteriophages were measured in triplicate. 


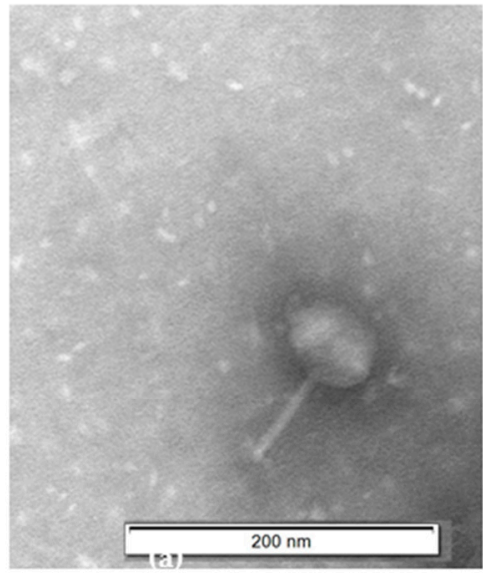

a)

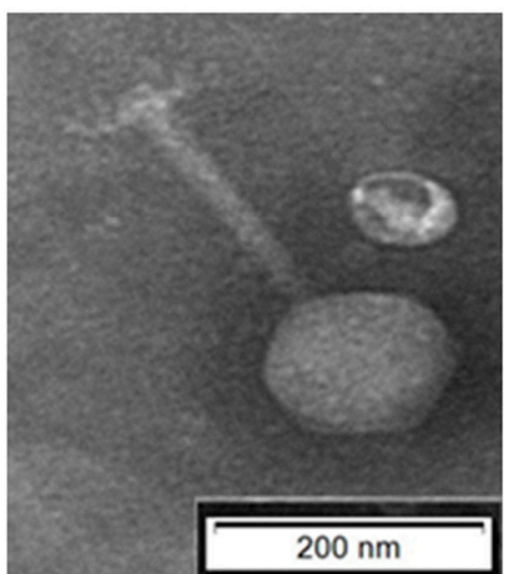

b)

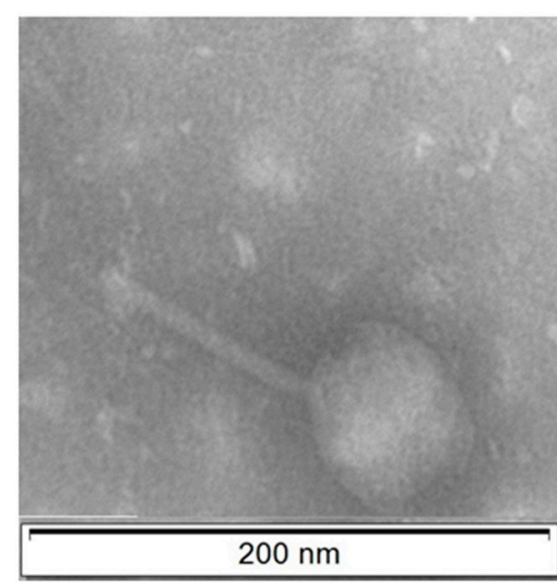

c)

Figure 2. Transmission electron micrographs of the Vibrio bacteriophages isolated in this study. (a) $\Phi-5$, (b) $\Phi-6$, (c) $\Phi-7$.

S1 nuclease and RNase A endonuclease digestion did not result in the production of fragments or degraded genomic DNA for any of the three isolated bacteriophages (results not shown), therefore, it was concluded that the nucleic acids of all bacteriophage isolates were double stranded DNA.

Using combined results from the TEM imaging and the digestions of genomic DNA, the $\Phi-5, \Phi-6$, and $\Phi-7$ were deduced to belong to the Myoviridae viral family (Table 2).

Genomes of $\Phi-5$ were aligned with toxic Vibrio genes: toxR, toxS gene (GenBank of AB372519.1), th gene (GenBank number of JQ929914.1), ctxA (GenBank number of FJ641050.1 and FJ641047.1), $t c p A$ and $t c p B$ (GenBank number of AF516340.1) using Blastn suite (Align Sequences Nucleotide BLAST) on https://blast.ncbi.nlm.nih.gov/Blast.cgi. The results revealed that there was no significant similarity between toxic genes located on the genome of $\Phi-5$. The annotation of the genomes of the $\Phi-5$ indicated that no integrase, transposase or repressor genes were present.

$\Phi-5$, which had strong lytic activity, was chosen for genome sequencing and it was found that $\Phi-5$ consisted of 238053 bp with an average GC content of $43.57 \%$. There was no ncRNA gene in the genome and a total of 217 ORFs were predicted for Vibrio $\Phi-5$ (Table 2). ORF 46, predicted as lysin, showed high level sequence similarity (95\%) to other previously described bacteriophage lysins. Putative thymidine kinase ( $92 \%$ identity), which is used in many antiviral drugs, was found in ORF 10 (Table S1).

Multiple sequence alignment revealed that $\Phi-5$ was closely related to Vibrio bacteriophage Aphrodite1 (GenBank number MG720308.1) with 95\% similarity and belonged to the genus Aphroditevirus. 
A proteomic tree (Figure 3) was produced based on TBLASTX genomic sequence of 26 bacteriophage genomes. The lengths of branches were logarithmically scaled. The tree was drawn by using the method of Nishimura et al. [31] (https://www.genome.jp/viptree/). Vibrio bacteriophage pTD1 and Vibrio bacteriophage Aphrodite1 has NCBI accession numbers of AP017972.1 and MG720308.1, respectively. Vibrio phage 2 and Vibrio phage 1 were isolated and stored at USC lab.

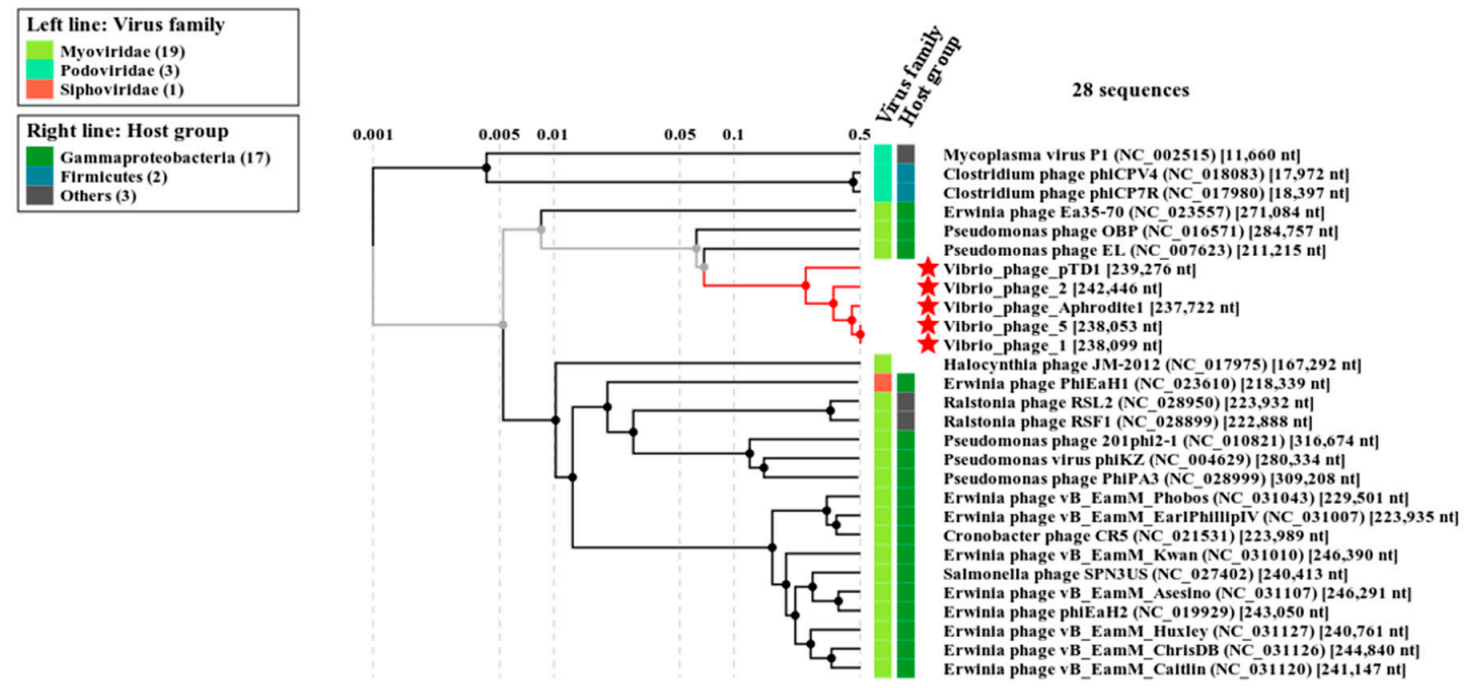

Figure 3. Bacteriophage proteomic tree.

Color bar (Figure 4) shows \% identity of TBLASTX. The comparative genomes were drawn by using a method of Nishimura et al. [31] (https://www.genome.jp/viptree/). Vibrio bacteriophage pTD1 and Vibrio bacteriophage Aphrodite1 has GenBank numbers of AP017972.1 and MG720308.1, respectively. Vibrio_phage_2 and Vibrio_phage_1 were isolated and stored at USC lab.
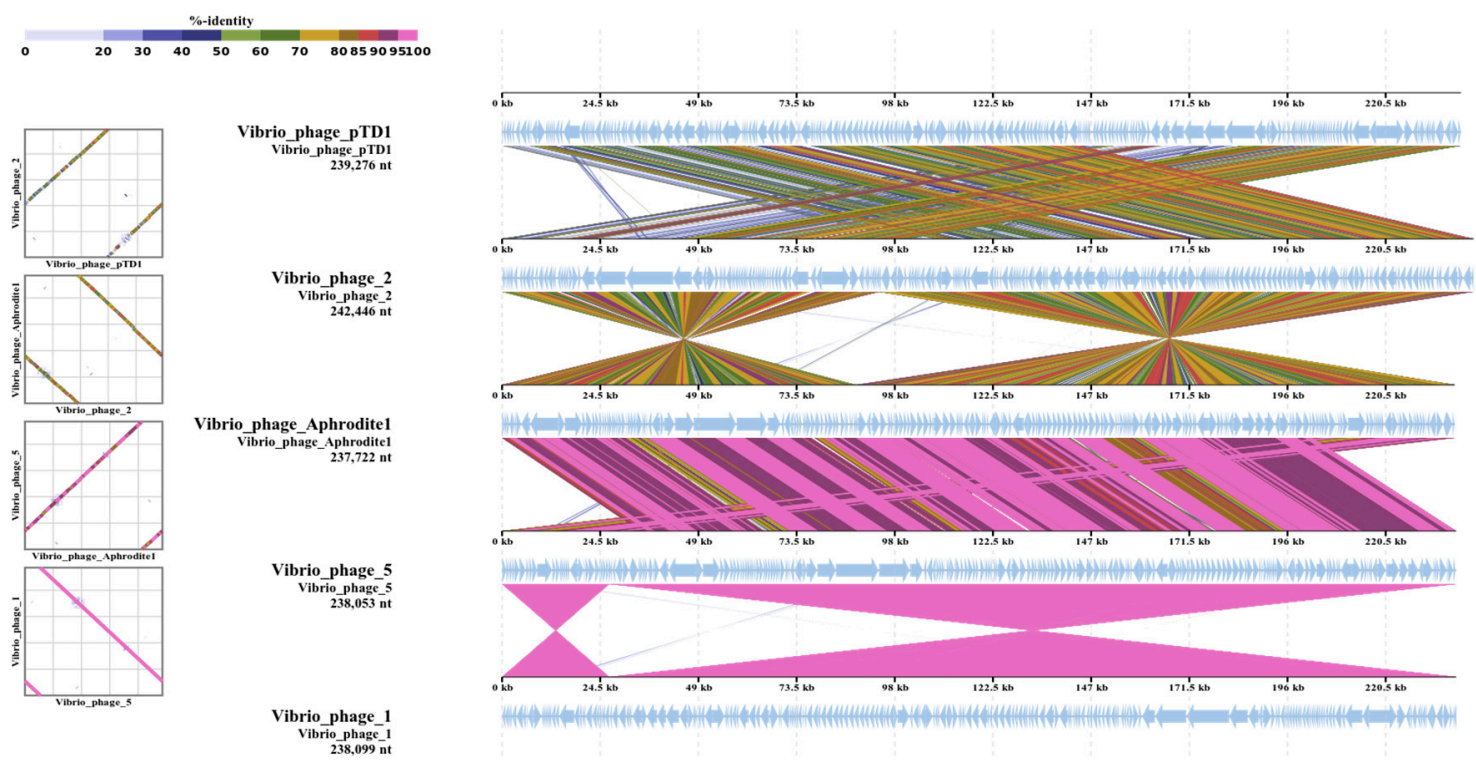

Figure 4. Analysis of the comparative genomes of the Vibrio bacteriophages.

\subsection{Host Range and Bacteriophage Lytic Ability}

$\Phi-6$ and $\Phi-7$ showed cross infectivity against $V$. harveyi (ATCC 14126) and $V$. alginolyticus (ATCC 17749), respectively (Table 3). Both isolated Vibrio spp. were susceptible to the bacteriophages. 
Table 3. Host range of the bacteriophage isolates determined against different Vibrio species.

\begin{tabular}{cccc}
\hline Vibrio spp. & Vibrio $\mathbf{\Phi - 5}$ & Vibrio $\mathbf{\Phi - 6}$ & Vibrio $\mathbf{\Phi - 7}$ \\
\hline USC-26004 & + & + & + \\
USC-26005 & $\mathrm{PH}$ & $\mathrm{PH}$ & $\mathrm{PH}$ \\
ATCC 14126 & - & + & - \\
ATCC 17749 & - & - & + \\
\hline "+": susceptible to bacteriophage lysis; “-": not susceptible to bacteriophage lysis; PH: propagation host.
\end{tabular}

In Luria-Bertani (LB) broth, concentrations of USC-26005 and USC-26004 increased gradually and reached average $\mathrm{OD}_{600 \mathrm{~nm}}$ values of 0.665 and 0.677 , respectively, during the $9 \mathrm{~h}$ incubation. Application of the Vibrio bacteriophages resulted in a high inhibition of bacterial growth. After $4 \mathrm{~h}$ for $\Phi-7$ treatment and $7 \mathrm{~h}$ for $\Phi-5$ treatment, bacterial growth recovered and reached much lower $\mathrm{OD}_{600 \mathrm{~nm}}$ values compared to the untreated control (Figure 5a).
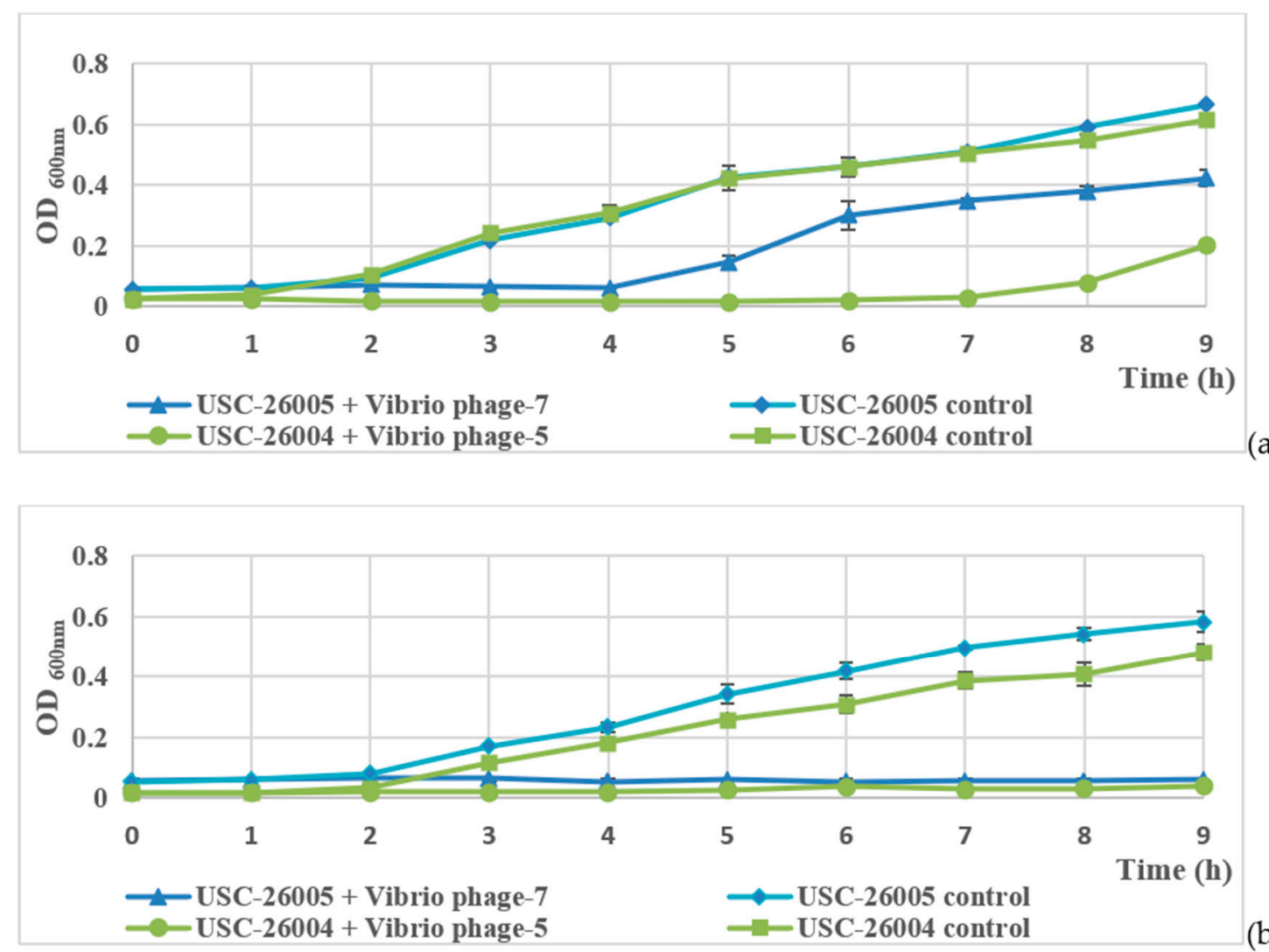

Figure 5. Inactivation of Vibrio spp. by the bacteriophages. Initial concentrations of $\Phi-6$ and $\Phi-7$ were $\times 10^{6} \mathrm{PFU} / \mathrm{mL}$. (a) LB broth: The initial concentrations of the isolates USC-26005 + $\Phi-7$, USC-26006 control, USC-2004 $+\Phi-5$ and USC-2004 control were $6.21 \pm 0.05 \times 10^{6}, 6.11 \pm 0.09 \times 10^{6}, 4.04 \pm 0.2$ $\times 10^{6}$ and $4.04 \pm 0.2 \times 10^{6} \mathrm{CFU} / \mathrm{mL}$, respectively. (b) A mixture of seawater and LB broth at a ratio of 3:1: The initial concentrations of the isolates USC-26005 + $\Phi-7$, USC-26006 control, USC-2004 + $\Phi-5$ and USC-2004 control were $6.24 \pm 0.1 \times 10^{6}, 5.92 \times 10^{6}, 3.62 \pm 0.05 \times 10^{6}$ and $3.59 \pm 0.12 \times 10^{6}$ $\mathrm{CFU} / \mathrm{mL}$, respectively.

In seawater, during infection with $\Phi-5$ and $\Phi-7$, the OD value of isolated USC-26005 and USC-26004 remained stable at around 0.06 and 0.02 . At the end of this experiment, the $\mathrm{OD}_{600 \mathrm{~nm}}$ value of USC-26005 control and USC-26004 control were at the highest levels of $0.584 \pm 0.032$ and $0.481 \pm 0.027$, respectively (Figure 5b). 


\subsection{Laboratory Trial of Bacteriophages Against Vibrio spp.}

There was no significant difference between control 1 and control $2(p>0.05)$, indicating that the bacteriophage cocktail did not affect oyster larval health. Mortality rate (\%) of larvae was $28.2 \pm 3.5$ in the bacteriophage treatment group, compared to $77.9 \pm 9.1$ in the bacterial treatment group after $24 \mathrm{~h}$ incubation (Figure 6).

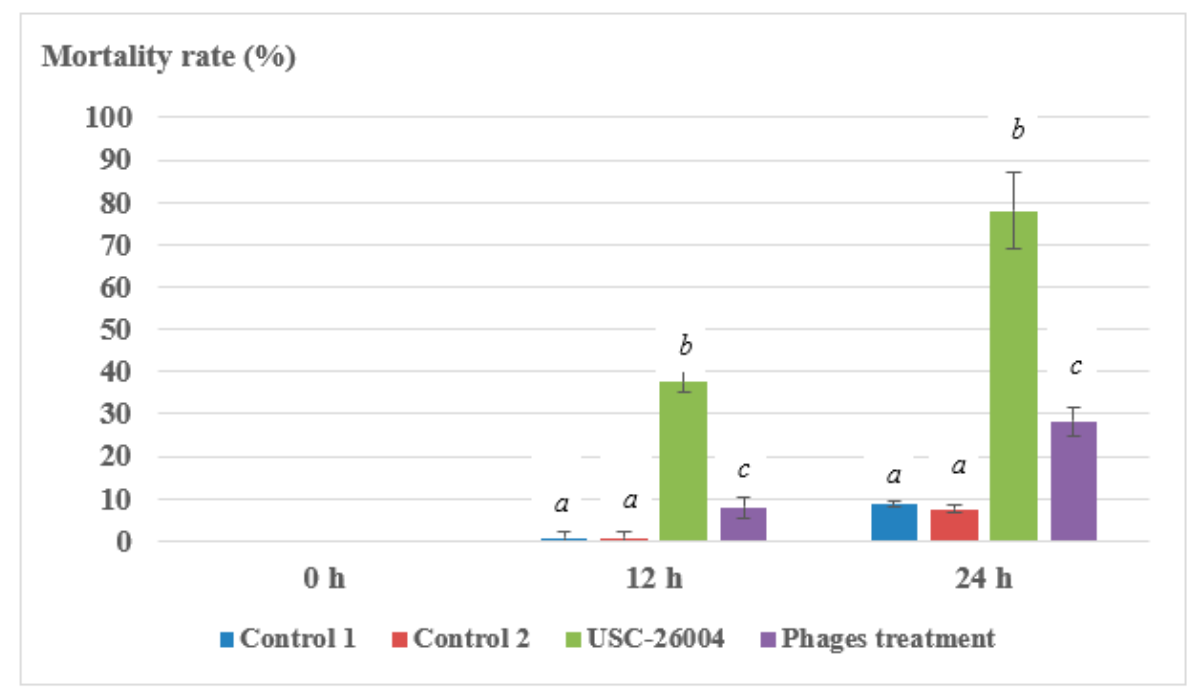

Figure 6. Mortality rate (\%) of oyster (Saccostrea glomerata) larvae when challenged with USC-26004 and treated with a bacteriophage cocktail. The bacteriophage cocktail was composed of a 1:1:1 mixture of the bacteriophages $\Phi-5, \Phi-6$ and $\Phi-7$. Control 1 (mixed phages cocktail), control 2 (larvae only with sterile seawater). Letters ( $a, b$, and $c)$ donate statistically significant differences between treatments versus time among the same types of applications.

\section{Discussion}

Several Vibrio spp., including V. alginolyticus, are considered to be the most significant aquaculture pathogens causing mortality of shellfish and crustaceans. Three antibiotics (oxytetracycline, chloramphenicol and oxolinic acid) are commonly used in aquaculture [2]. Watts et al. [32] pointed out that $90 \%$ of bacteria isolated from seawater were resistant to at least one antibiotic and $20 \%$ of bacteria were resistant to five or more antibiotics. Kang et al. [14] reported on the pathogenic $V$. alginolyticus that caused severe infection in shellfish in coastal areas of Korea where all of 15 isolates tested were resistant to vancomycin and ampicillin and 33\% of Vibrio isolates showed multiple antimicrobial resistance to at least three antibiotics. Even though the use of antibiotics in some developed countries such as Japan, North America, and Europe is strictly regulated or banned, there is a lack of regulation on antibiotics use in developing nations where $90 \%$ of world aquaculture production occurs. This results in high variability in the use of antibiotics in different regions and the potential accumulation of antibiotic residues in the produce. Antibiotic residues in farmed salmon for example, ranged from 0.02- $0.39 \mathrm{~g} /$ ton in Scotland and Norway, to $660 \mathrm{~g} /$ ton in Chile [32]. In line with the above information, the most pathogenic isolate in the present study was USC-26004 which also displayed antibiotic resistant properties. Antibiotic resistance was also observed for Vibrio isolates similar to those observed by Kang et al. [14] and Karunasagar et al. [12], indicating that antibiotic resistant Vibrio species may have increasing prevalence in the environment. Antibiotic resistance also occurs in aquaculture systems that are considered "hotspots for antibiotic resistance genes" where recombination and gene exchange can easily occur [32]. As a result, bacteriophage control that also eliminates the possibility of antibiotic resistant bacteria, presents an effective potential control strategy.

Ackermann [33] reported that from a total of 227 tailed Vibrio bacteriophages, 67 (29.5\%) and $86(37.9 \%)$ belonged to the Siphoviridae and Myoviridae viral families, respectively. In the present 
study, the Vibrio bacteriophage isolates were also classified into the Myoviridae viral family. Moreover, the sizes of the Vibrio bacteriophages corresponded to those reported by Ackermann [34], where capsid and tail size of tailed bacteriophages were from 30 to 160 and 10 to $800 \mathrm{~nm}$, respectively. The three bacteriophage genomes could not be digested by S1 nuclease or RNase A, confirming that they were all double stranded DNA bacteriophages, and again, these findings were consistent with results from Sasikala and Srinivasan [35]. The genome of $\Phi-5$ was relatively large, compared to PVA1 Vibrio alginolyticus bacteriophage, in which the genomic sequence contained 41529 bp with a GC content of $43.7 \%$ [36].

For acceptance of the use of bacteriophages as biological control agents in aquaculture, better understanding of the genetics and the biology of bacteriophages is required. One of the potential obstacles can be related to temperate bacteriophages, that can transduce virulence factors to host bacteria [37]. Many researchers have reported that bacteriophages can encode toxins or other toxicity factors which could increase the virulence of their host bacteria. This obstacle could be removed by selecting virulent bacteriophages. Moreover, in order to be an effective biological control candidate, the genome of selected bacteriophages should not contain any of the known virulence-associated or antibiotic-resistance genes [37]. Therefore, bacteriophage genome analysis has to be carried out to determine that none of the putative proteins would have significant similarities to hypothetical factors or genes known to play roles in bacterial pathogenicity. The annotation of the genome of $\Phi-5$ indicated that no integrase, transposase or repressor genes were present in the genome. Therefore, the truly lytic nature of $\Phi-5$ implied that it is a suitable candidate for biological control of $V$. alginolyticus (USC 26001, USC 26004, ATCC 17749). Another potential obstacle to the use of bacteriophages as biocontrol agents is to ensure that they do not perform generalized or specialized transduction [38]. Based on the genome analysis and the subsequent growth experiments, it was concluded that the bacteriophages described in this paper were unlikely to be involved in the specialized transduction of host bacterial DNA. The GC content of $\Phi-1$ was very close to that for bacteriophage V. alginolyticus Vp 670 (GC content of 43.4\%) [39] with no ncRNA gene in the genome [38]. Bacteriophages with tails and a dsDNA genome larger than $200 \mathrm{~kb}$ are classified as Jumbo bacteriophage [40]. $\Phi-5$ is a jumbo bacteriophage. Similar to other Jumbo phages, which are often isolated from aquatic environments, they mostly infect Gram-negative host bacteria (95.6\%) [40].

Until now, 578 Vibrio bacteriophage genomes have been sequenced (http://millardlab.org/ bioinformatics/bacteriophage-genomes/phage-genomes-april-2019/) and the data obtained have paved the way to new approaches relating to the utilization of Vibrio bacteriophage lysins as potential antimicrobial agents. However, due to the outer-membrane protection of Gram-negative bacteria, lysins can only act effectively against Gram-positive bacteria and they work less effectively against Gram-negative bacteria. Lysins might be more effective against Gram-negative bacteria if the bacteria were pretreated with the outer membrane proteins $\left(\mathrm{CHCl}_{3}\right.$, EDTA or Triton X-100) [41-44]. In order to apply in aquaculture settings, large volume and cost-effective scale-up of lysin production is required. These factors are currently significant obstacles against cost-effective implementation of the use of lysins for disease control in aquaculture. Therefore, in this study, phage lysin was not investigated.

Further studies could be conducted to determine whether or not bacteriophage treatments would be effective in aquaculture facilities. Moreover, the performance of bacteriophages under the range of environmental conditions that might be experienced in aquaculture systems needs to be investigated to enable generation of an improved understanding of appropriate and sub-optimal conditions under which bacteriophage treatment might be successfully applied. Further research should also be carried out on bacteriophage-resistant bacteria to confirm that mutant bacteria are not likely to be pathogenic [45]. 


\section{Materials and Methods}

\subsection{Isolation and Characterization of Vibrio spp. Isolated from Oyster Hatcheries}

Vibrio spp. were isolated from water within larval culture tanks at an oyster hatchery (Port Stephens Fisheries Institute, New South Wales, Australia), by concentrating samples from the cultures on Whatman filter papers (Merck, Macquarie Park NSW, Australia) followed by an enrichment. Isolates were then identified using conventional biochemical tests in the form of commercial kits API ${ }^{\circledR}$ 20NE (BioMerieux, Durham, NC 27712, USA) (https://www.biomerieux-usa.com/clinical/api). After incubation, colony morphology on the agar plates was examined, isolations were conducted, and the resulting pure bacterial cultures were stored at $-80{ }^{\circ} \mathrm{C}$ in the DMB supplemented with $15 \%$ glycerol.

The DNA of isolated bacteria was extracted according to the manufacturer's instructions, using the GenElute bacterial genomic DNA kit (Merck, Macquarie Park NSW, Australia). Universal primers U1492R and Bac27F [46] were used to amplify the partial 16S rDNA gene, using thermal cycles, based on the method of Pang et al. [47]. The resulting PCR products were sequenced by the Macrogen Company in the Republic of Korea (http://www.macrogen.com/en/main/index.php) and the sequences were subsequently analyzed using BLAST analysis (https://blast.ncbi.nlm.nih.gov). The resulting sequence of USC-26004 was deposited in Genbank (accession number of MK334309).

The disk diffusion method [48] on Müller-Hinton agar (Thermo Scientific, Scoresby, Vic, Australia) was used to determine the antibiotic resistance patterns of the Vibrio isolates against the following antimicrobials: aztreonam $(30 \mu \mathrm{g})$, tetracycline $(30 \mu \mathrm{g})$, nalidixic acid $(30 \mu \mathrm{g})$, cefoxitin $(30 \mu \mathrm{g})$, erythromycin $(15 \mu \mathrm{g})$, gentamicin $(10 \mu \mathrm{g})$, streptomycin $(10 \mu \mathrm{g})$, ampicillin $(10 \mu \mathrm{g})$, imipenem $(10 \mu \mathrm{g})$, and penicillin (10 units) (Edwards Group Pty. Ltd., NSW, Australia). Plates were incubated for $24 \mathrm{~h}$ at $37^{\circ} \mathrm{C}$. Because no breakpoints are defined for Vibrio, the zone diameter interpretive standards for the Enterobacteriaceae [48] was used in this study and the E. coli strain (ATCC 25922) was used as the control during the test.

\subsection{Pathogenicity Test and Prophage Induction}

All isolated Vibrio spp., V. harveyi (ATCC 14126), and V. alginolyticus (ATCC 17749) were tested for their possible pathogenicity against oyster larvae using the method described by Prado et al. [30]. Healthy Sydney rock oyster (Saccostrea glomerata) larvae (200 $\mu \mathrm{m}$ size antero-posterior shell size) were used at a density of 8-12 larvae/mL in $2.5 \mathrm{~mL}$ wells (total of 24-30 larvae per well) and the final concentration of Vibrio spp. administered to larval cultures was $\sim 10^{5} \mathrm{CFU} / \mathrm{mL}$. Mortalities lower than $10 \%$ indicated that the bacteria were not pathogenic [30]. All tested groups were performed in triplicate.

The prophage induction test of Vibrio spp. was carried out as described by Clokie and Kropinski [49].

\subsection{Isolation and Characterization of Bacteriophages}

Bacteriophage isolation and genome sequencing was carried out as described by Clokie and Kropinski [49].

Rectangular proteomic tree and genomic alignment were created by using method of Nishimura et al. [31].

Genome of $\Phi-5$ was aligned with toxic Vibrio genes: toxR, toxS gene (GenBank of AB372519.1), tlh gene (GenBank number of JQ929914.1), ctxA (GenBank number of FJ641050.1 and FJ641047.1), tcpA and tcpB (GenBank number of AF516340.1) using Blastn suite (Align Sequences Nucleotide BLAST) on https://blast.ncbi.nlm.nih.gov/Blast.cgi.

The GenBank numbers of $\Phi-1$ (or Vibrio phage USC-1) and $\Phi-5$ were MK905543 and MK358448, respectively. 


\subsection{Bacteriophage Lytic Activity}

Vibrio species were streaked onto Tryptone Soya Agar with 1.5\% salt agar (TSAS) and incubated at $28{ }^{\circ} \mathrm{C}$ overnight, then harvested onto LB broth (Thermo Fisher Scientific, Scoresby, VIC, Australia). An aliquot $(6 \mathrm{~mL})$ of the Vibrio spp. in LB broth $\left(\sim 10^{6} \mathrm{CFU} / \mathrm{mL}\right)$ was added into $2 \mathrm{~mL}$ of a bacteriophage solution (concentration of $\sim 10^{6}$ to $\sim 10^{8} \mathrm{PFU} / \mathrm{mL}$ ) and incubated at $28^{\circ} \mathrm{C}$ and $150 \mathrm{rpm}$. A control using only Vibrio spp. was also included in the test. Samples were removed from the broth every hour for $9 \mathrm{~h}$ and the optical density of the cells measured at $600 \mathrm{~nm}$. A similar experiment to that as detailed above was carried out with the exception that the LB broth was replaced with sterile seawater to measure bacteriophage lytic activity in a mixture of sterile seawater and LB broth at a ratio of 3:1. All tests were performed in triplicate.

\subsection{Bacteriophage Control of Vibrio spp. with Oyster Larvae}

Vibrio sp. (USC-26004) was used for challenge tests in 12-well sterile tissue cell-culture plates (Thermo Fisher Scientific, Scoresby, VIC, Australia) [30,50]. Oyster (S. glomerata) larvae with an antero-posterior shell size of around $200 \mu \mathrm{m}$, were placed at a stocking density of 8-12 larvae/mL into each $2.5 \mathrm{~mL}$ well (total of 24-30 larvae per well). A bacteriophage cocktail composed of a 1:1:1 mixture of the bacteriophages $\Phi-5, \Phi-6$ and $\Phi-7$ was prepared. The challenge included a positive control group (larvae with exposure to USC-26004), a bacteriophage control 1 (mixed phages cocktail), control 2 (larvae only with sterile seawater) and a bacteriophage treated group. An aliquot $(0.1 \mathrm{~mL})$ of mixed bacteriophage cocktail (final concentration of $10^{8} \mathrm{PFU} / \mathrm{mL}$ ) was first added at $0 \mathrm{~h}$ and then added with another $0.1 \mathrm{~mL}$ of the same bacteriophage suspension after $12 \mathrm{~h}$. Vibrio USC-26004 was added to give a final concentration of $10^{5} \mathrm{CFU} / \mathrm{mL}$ in the well. The challenges were run for a period of $24 \mathrm{~h}$. Larval mortality was determined every $12 \mathrm{~h}$ using a light microscope $(4 \times-10 \times)$ and mortality was characterized by the lack of identifiable movement of larval tissues. Larvae were fed every $12 \mathrm{~h}$ during the challenge test with live microalgae (Tetraselmis chuii, Dunaliella tertiolecta and Nannochloropsis oculata) following standard culture procedure for Sydney rock oyster larvae(https://www.dpi.nsw.gov. au/_data/assets/pdf_file/0014/261041/Output-1084_Hatchery-Manual_FORMATTED.pdf). All tested groups were performed in triplicate.

\subsection{Statistical Analysis}

Single factor ANOVA was used to test for differences in larval mortality rates in treatments with and without the bacteriophage suspension. Data were analyzed using SPSS Statistics 20 software (IBM SPSS Statistics 20, USA) (https://www-01.ibm.com/support/docview.wss?uid=swg24029274) and difference was considered significant at $p<0.05$.

\section{Conclusions}

A bacteriophage cocktail composed of three newly isolated bacteriophages investigated in this study successfully reduced levels of Vibrio spp. contamination and improved the survival of oyster larvae exposed to pathogenic Vibrio spp. On this basis, bacteriophage treatment may be regarded as having potential as an effective biocontrol agent that prevents the onset of vibriosis in oyster hatcheries and, potentially, in other aquaculture settings. The genome of $\Phi-5$ was found to contain lysin genes. Findings presented increase our understanding of bacteriophage biology, genomics, and bacteriophage lysins, and they highlight the potential of bacteriophage biocontrol as an alternative treatment for vibriosis in aquaculture facilities.

Supplementary Materials: The following are available online at http://www.mdpi.com/2079-6382/9/7/415/s1, Table S1: Predicted ORFs for $\Phi-5$ and homology to proteins. 
Author Contributions: T.S.L. conducted experiments, analyzed the data and drafted the manuscript. S.V.V. assisted with the statistical data analysis. D.I.K., W.O. and P.C.S. provided supervision with the experimental design, interpretation of data/analyses and oversaw the preparation of the manuscript. All authors have read and agreed to the published version of the manuscript.

Funding: This research received no external funding.

Acknowledgments: Tuan Son Le would like to acknowledge MOET-VIED/USC PhD scholarship. Authors would like to thank Global Catalogue of Microorganisms (GCM) [51] project (project code: F18FTSNCKF2200) for the phage genome analysis. Australian provisional patent application number 2019900622 including information contained in this article was submitted on the 27th of February 2019.

Conflicts of Interest: The authors declare no conflict of interest.

\section{References}

1. FAO. The State of World Fisheries and Aquaculture 2018-Meeting the Sustainable Development Goals; FAO: Rome, Italy, 2018.

2. Lucas, J.S. Bivalve molluscs. In Aquaculture: Farming Aquatic Animals and Plants, 3rd ed.; Lucas, J.S., Southgate, P.C., Eds.; Wiley-Blackwell Publishing Ltd.: Oxford, UK, 2019; pp. 541-566.

3. Elston, R.A.; Hasegawa, H.; Humphrey, K.L.; Polyak, I.K.; Häse, C.C. Re-emergence of Vibrio tubiashii in bivalve shellfish aquaculture: Severity, environmental drivers, geographic extent and management. Dis. Aquat. Organ. 2008, 82, 119-134. [CrossRef] [PubMed]

4. Romalde, J.; Barja, J. Bacteria in molluscs: Good and bad guys. Curr. Res. Technol. Educ. Top. Appl. Microbiol. Microb. Biotechnol. 2010, 1, 136-147.

5. Tubiash, H.S.; Chanley, P.E.; Leifson, E. Bacillary necrosis, a disease of larval and juvenile bivalve mollusks I. etiology and epizootiology. J. Bacteriol. 1965, 90, 1036-1044. [CrossRef] [PubMed]

6. Sugumar, G.; Nakai, T.; Hirata, Y.; Matsubara, D.; Muroga, K. Vibrio splendidus biovar II as the causative agent of bacillary necrosis of Japanese oyster Crassostrea gigas larvae. Dis. Aquat. Organ. 1998, 33, 111-118. [CrossRef]

7. Prado, S.; Dubert, J.; Romalde, J.L.; Toranzo, A.E.; Barja, J.L. Vibrio ostreicida sp. nov., a new pathogen of bivalve larvae. Int. J. Syst. Evol. Microbiol. 2014, 64, 1641-1646. [CrossRef]

8. Dubert, J.; Nelson, D.R.; Spinard, E.J.; Kessner, L.; Gomez-Chiarri, M.; da Costa, F.; Prado, S.; Barja, J.L. Following the infection process of vibriosis in manila clam (Ruditapes philippinarum) larvae through gfp-tagged pathogenic Vibrio species. J. Invertebr. Pathol. 2016, 133, 27-33. [CrossRef]

9. Southgate, P.C. Foods and feeding. In Aquaculture: Farming Aquatic Animals and Plants, 3rd ed.; Lucas, J.S., Southgate, P.C., Eds.; Wiley-Blackwell Publishing Ltd.: Oxford, UK, 2019; pp. 188-213.

10. Dubert, J.; Barja, J.L.; Romalde, J.L. New insights into pathogenic Vibrios affecting bivalves in hatcheries: Present and future prospects. Front. Microbiol. 2017, 8, 762. [CrossRef]

11. Defoirdt, T.; Boon, N.; Sorgeloos, P.; Verstraete, W.; Bossier, P. Alternatives to antibiotics to control bacterial infections: Luminescent vibriosis in aquaculture as an example. Trends Biotechnol. 2007, 25, 472-479. [CrossRef]

12. Karunasagar, I.; Pai, R.; Malathi, G.; Karunasagar, I. Mass mortality of Penaeus monodon larvae due to antibiotic-resistant Vibrio harveyi infection. Aquaculture 1994, 128, 203-209. [CrossRef]

13. Igbinosa, E.O. Detection and antimicrobial resistance of Vibrio isolates in aquaculture environments: Implications for public health. Microb. Drug Resist. 2016, 22, 238-245. [CrossRef]

14. Kang, C.-H.; Shin, Y.; Jang, S.; Jung, Y.; So, J.-S. Antimicrobial susceptibility of Vibrio alginolyticus isolated from oyster in Korea. Environ. Sci. Pollut. R. 2016, 23, 21106-21112. [CrossRef] [PubMed]

15. Wang, Y.; Barton, M.; Elliott, L.; Li, X.; Abraham, S.; O’Dea, M.; Munro, J. Bacteriophage therapy for the control of Vibrio harveyi in Greenlip abalone (Haliotis laevigata). Aquaculture 2017, 473, 251-258. [CrossRef]

16. Rong, R.; Lin, H.; Wang, J.; Khan, M.N.; Li, M. Reductions of Vibrio parahaemolyticus in oysters after bacteriophage application during depuration. Aquaculture 2014, 418, 171-176. [CrossRef]

17. Le, T.S.; Nguyen, T.H.; Vo, H.P.; Doan, V.C.; Nguyen, H.L.; Tran, M.T.; Tran, T.T.; Southgate, P.C.; Kurtböke, D.İ. Protective effects of bacteriophages against Aeromonas hydrophila causing motile aeromonas septicemia (MAS) in striped catfish. Antibiotics 2018, 7, 16. [CrossRef] [PubMed] 
18. Kim, H.J.; Jun, J.W.; Giri, S.S.; Chi, C.; Yun, S.; Kim, S.G.; Oh, W.T. Application of the bacteriophage pVco-14 to prevent Vibrio coralliilyticus infection in Pacific oyster (Crassostrea gigas) larvae. J. Invertebr. Pathol. 2019, 167, 107244. [CrossRef] [PubMed]

19. Wang, I.-N.; Smith, D.L.; Young, R. Holins: The protein clocks of bacteriophage infections. Annu. Rev. Microbiol. 2000, 54, 799-825. [CrossRef]

20. Oliveira, H.; Melo, L.D.; Santos, S.B.; Nóbrega, F.L.; Ferreira, E.C.; Cerca, N.; Azeredo, J.; Kluskens, L.D. Molecular aspects and comparative genomics of bacteriophage endolysins. J. Virol. 2013, 87, 4558-4570. [CrossRef]

21. Fischetti, V. Development of phage lysins as novel therapeutics: A historical perspective. Viruses 2018, 10, 310. [CrossRef]

22. Witzenrath, M.; Schmeck, B.; Doehn, J.M.; Tschernig, T.; Zahlten, J.; Loeffler, J.M.; Zemlin, M.; Müller, H.; Gutbier, B.; Schütte, H. Systemic use of the endolysin cpl-1 rescues mice with fatal pneumococcal pneumonia. Crit. Care Med. 2009, 37, 642-649. [CrossRef]

23. Resch, G.; Moreillon, P.; Fischetti, V.A. A stable phage lysin (cpl-1) dimer with increased antipneumococcal activity and decreased plasma clearance. Int. J. Antimicrob. Agents 2011, 38, 516-521. [CrossRef]

24. Gilmer, D.B.; Schmitz, J.E.; Euler, C.W.; Fischetti, V.A. Novel bacteriophage lysin with broad lytic activity protects against mixed infection by Streptococcus pyogenes and methicillin-resistant Staphylococcus aureus. Antimicrob. Agents Chemoter. 2013, 57, 2743-2750. [CrossRef]

25. Wang, Q.; Euler, C.W.; Delaune, A.; Fischetti, V.A. Using a novel lysin to help control Clostridium difficile infections. Antimicrob. Agents Chemoter. 2015, 59, 7447-7457. [CrossRef] [PubMed]

26. Larpin, Y.; Oechslin, F.; Moreillon, P.; Resch, G.; Entenza, J.M.; Mancini, S. In vitro characterization of plye146, a novel phage lysin that targets gram-negative bacteria. PLoS ONE 2018, 13, e0192507. [CrossRef]

27. Lood, R.; Winer, B.Y.; Pelzek, A.J.; Diez-Martinez, R.; Thandar, M.; Euler, C.W.; Schuch, R.; Fischetti, V.A. Novel phage lysin capable of killing the multidrug-resistant gram-negative bacterium Acinetobacter baumannii in a mouse bacteremia model. Antimicrob. Agents Chemoter. 2015, 59, 1983-1991. [CrossRef] [PubMed]

28. Oliveira, H.; Vilas Boas, D.; Mesnage, S.; Kluskens, L.D.; Lavigne, R.; Sillankorva, S.; Secundo, F.; Azeredo, J. Structural and enzymatic characterization of abgp46, a novel phage endolysin with broad anti-gram-negative bacterial activity. Front. Microbiol. 2016, 7, 208. [CrossRef] [PubMed]

29. Chibani-Chennoufi, S.; Bruttin, A.; Dillmann, M.-L.; Brüssow, H. Phage-host interaction: An ecological perspective. J. Bacteriol. 2004, 186, 3677-3686. [CrossRef] [PubMed]

30. Prado, S.; Romalde, J.L.; Montes, J.; Barja, J.L. Pathogenic bacteria isolated from disease outbreaks in shellfish hatcheries. First description of Vibrio neptunius as an oyster pathogen. Dis. Aquat. Organ. 2005, 67, $209-215$. [CrossRef]

31. Nishimura, Y.; Yoshida, T.; Kuronishi, M.; Uehara, H.; Ogata, H.; Goto, S.J.B. Viptree: The viral proteomic tree server. Bioinformatics 2017, 33, 2379-2380. [CrossRef]

32. Watts, J.E.; Schreier, H.J.; Lanska, L.; Hale, M.S. The rising tide of antimicrobial resistance in aquaculture: Sources, sinks and solutions. Mar. Drugs 2017, 15, 158. [CrossRef]

33. Ackermann, H.-W. 5500 phages examined in the electron microscope. Arch. Virol. 2007, 152, $227-243$. [CrossRef]

34. Ackermann, H.W. Bacteriophage classification. In Bacteriophages: Biology and Applications; Kutter, E., Sulakvelidze, A., Eds.; CRC Press: Boca Raton, FL, USA, 2005; pp. 67-89.

35. Sasikala, D.; Srinivasan, P. Characterization of potential lytic bacteriophage against Vibrio alginolyticus and its therapeutic implications on biofilm dispersal. Microb. Pathog. 2016, 101, 24-35. [CrossRef] [PubMed]

36. Zhang, J.; Cao, Z.; Xu, Y.; Li, X.; Li, H.; Wu, F.; Wang, L.; Cao, F.; Li, Z.; Li, S. Complete genomic sequence of the Vibrio alginolyticus lytic bacteriophage pva1. Arch. Virol. 2014, 159, 3447-3451. [CrossRef] [PubMed]

37. Carrias, A. Evaluation of biological agents for controlling enteric septicemia of catfish. Ph.D. Thesis, Auburn University, Auburn, AL, USA, 2011.

38. Jacquemot, L.; Bettarel, Y.; Monjol, J.; Corre, E.; Halary, S.; Desnues, C.; Bouvier, T.; Ferrier-Pagès, C.; Baudoux, A.C. Therapeutic potential of a new jumbo phage that infects Vibrio coralliilyticus, a widespread coral pathogen. Front. Microbiol. 2018, 9, 2501. [CrossRef] [PubMed]

39. Luo, P.; Yun, L.; Li, Y.; Tian, Y.; Liu, Q.; Huang, W.; Hu, C. Complete genomic sequence of the Vibrio alginolyticus bacteriophage Vp670 and characterization of the lysis-related genes, cwlQ and hola. BMC Genom. 2018, 19, 741. [CrossRef] 
40. Yuan, Y.; Gao, M. Jumbo bacteriophages: An overview. Front. Microbiol. 2017, 8, 403. [CrossRef]

41. Guo, M.; Feng, C.; Ren, J.; Zhuang, X.; Zhang, Y.; Zhu, Y.; Dong, K.; He, P.; Guo, X.; Qin, J. A novel antimicrobial endolysin, lyspa26, against Pseudomonas aeruginosa. Front. Micriobiol. 2017, 8, 293. [CrossRef]

42. Elliman, J. Bacteriophage of Burkholderia pseudomallei; Friend or Foe? Ph.D. Thesis, James Cook University, Queensland, Australia, 2006.

43. Hertwig, S.; Bockelmann, W.; Teuber, M. Purification and characterization of the lytic activity induced by the prolate-headed bacteriophage p001 in Lactococcus lactis. J. Appl. Microbiol. 1997, 82, 233-239. [CrossRef]

44. Pastagia, M.; Schuch, R.; Fischetti, V.A.; Huang, D.B. Lysins: The arrival of pathogen-directed anti-infectives. J. Med. Microbiol. 2013, 62, 1506-1516. [CrossRef]

45. Crothers-Stomps, C.; Høj, L.; Bourne, D.; Hall, M.; Owens, L. Isolation of lytic bacteriophage against Vibrio harveyi. J. Appl. Microbiol. 2010, 108, 1744-1750. [CrossRef]

46. Weisburg, W.G.; Barns, S.M.; Pelletier, D.A.; Lane, D.J. 16s ribosomal DNA amplification for phylogenetic study. J. Bacteriol. 1991, 173, 697-703. [CrossRef]

47. Pang, L.; Zhang, X.H.; Zhong, Y.; Chen, J.; Li, Y.; Austin, B. Identification of Vibrio harveyi using PCR amplification of the toxr gene. Lett. Appl. Microbiol. 2006, 43, 249-255. [CrossRef] [PubMed]

48. Clinical and Laboratory Standards Institute. Performance Standards for Antimicrobial Susceptibility Testing: Twentieth Informational Supplement m100-s20; Clinical and Laboratory Standards Institute: Wayne, PA, USA, 2010.

49. Clokie, M.R.; Kropinski, A. Methods and Protocols, Volume 1: Isolation, Characterization, and Interactions; Humana Press: Totowa, NJ, USA, 2009.

50. Estes, R.M.; Friedman, C.S.; Elston, R.A.; Herwig, R.P. Pathogenicity testing of shellfish hatchery bacterial isolates on pacific oyster Crassostrea gigas larvae. Dis. Aquat. Organ. 2004, 58, 223-230. [CrossRef] [PubMed]

51. Wu, L.; Ma, J. The global catalogue of microorganisms (GCM) 10k type strain sequencing project: Providing services to taxonomists for standard genome sequencing and annotation. Int. J. Syst. Evol. Microbiol. 2019, 69, 895-898. [CrossRef] [PubMed]

(C) 2020 by the authors. Licensee MDPI, Basel, Switzerland. This article is an open access article distributed under the terms and conditions of the Creative Commons Attribution (CC BY) license (http://creativecommons.org/licenses/by/4.0/). 